\title{
Basic special needs and related approaches - mainly medical - for patients following moderate/severe traumatic brain injury
}

\author{
Cristina POPESCU ${ }^{1,2}$, Aurelian ANGHELESCU ${ }^{1,2}$, Cristina DAIA ${ }^{1,2}$, Gelu ONOSE ON, $^{1,2}$ \\ ${ }^{1}$ Physical (Neural-Muscular) and Rehabilitation Medicine Clinic Division, \\ „Bagdasar-Arseni“ Teaching Emergency Hospital, Bucharest, Romania \\ ${ }^{2}$ "Carol Davila“ University of Medicine and Pharmacy, Bucharest, Romania
}

\begin{abstract}
Traumatic brain injury (TBI) may affect almost every aspect of a person's life. The relationships with family and friends, their roles and responsibilities, all, will require an adjustment period, preferably to be achieved one step at a time, with training and guidance of a specialized health care team.

Consequently, such neurologically severe impaired patients have almost always including long term needs, which are quasi-permanent or permanent so endured for a life time. The only way these special needs can be fulfilled or - at best made unnecessary - is the discovery of the way to cure the central nervous system lesions. Because medicine is unfortunately not there yet, this article will review the respective specific numerous long-term needs that may be met by the tools medicine has to offer today, aiming to improve health care in hospital units with rehabilitation profile and to provide ongoing guideline constructs for patients with TBI and their families, after discharge too, aiming at an as complete as possible family social and professional reintegration.

Post TBI evolution is influenced by a variety of factors making patients with brain injuries care a complicated and sustained process, the improvements being undoubtedly influenced by their multimodal way to be approached within interdisciplinary teams, applying individualized rehabilitation programs - all in purpose to reach a quasi-normal life or as close to its sense.
\end{abstract}

Keywords: long-term needs, disability, traumatic brain injury

\section{BACKGROUND}

In the last decades there have been a number of achievements in some health assistance subfields, including emergency medicine, by improving the integrated management system of specialized related services intervening in trau$\mathrm{ma}$ /polytrauma-including neurotrauma - which led paradoxically, to a continuous increase in the number of people - survivors after various life threatening conditions - but with, including severe remaining, disabilities.

Disability is not only a health issue, at the same time accounting for a complex expression that reflects interaction between functions of a person body and aspects of society. To over- 
come the hurdles experienced by disabled people impose interventions to remove also any social and environmental obstacles. (1)

A point which is worth mentioning is that patients, of all ages, following moderate or severe $\mathrm{TBI}$, with different consequent disabilities are also "hyper-chronic" in their long term evolution. (2)

It is well known, that people with chronic pathology - especially with life threatening or impairment(s)/disability(es) ingeneration potential - have to deal with more problems than healthy individuals. Every trivial thing that can be done easily by a normal person can become a challenge for chronically impaired patients. These types of individuals are called „persons with special needs" (medically and/or non-medically). This term includes a wide spread variety of "needs" regarding specific conditions that may vary from severe physical to mental, cognitive, emotional impairments, their disabilities ranging from speech disorder to quadriplegia. (3) Thus, from a health assistance point of view, these "long term needs" can become permanent and continuous up to 24 hours daily nursing assistance. (4)

Post TBI patients are individuals with acquired nervous system lesions - evolving or non-evolving - which have lead to more or less extended impairments. These lesions can affect: voluntary motricity (also muscle tone and/ or tissue trophicity), coordination, balance, sphincters`control, sensitive, sensorial - especially for some brain ailments, swallowing, cognitive/consciousness, communication ability and respectively emotional/behavioral aspects but also there can occur endocrine dysfunctions and various forms of pain (hyperalgesia, hyperesthesia, hyperpathia or paresthesia, borrowing characters of neuropathic pain; sometimes pain is non neuropathic, it is due to the lower urinary tract infection or it may be induced by spasticity, immobilization, lying, vicious postures).

More specifically from the medical point of view, patients with special needs imply the presence of: cognitive and physical disabilities, of „mental illness", communication disorders and/ or hearing, vision usually associated with pour literacy level, „poverty and aging“. (4)

At the same time, besides the long-term needs exposed may be added: development assistance/habilitation and possibly custom school education - for some cases in children and teenagers -, aspects of rehabilitation also in the professional domain (ergo-/occupational therapy) in adults - entailing specific infrastructure including with access to appropriate assistive devices, if applicable -, economic (mainly regarding fiscal dedicated regulations) support for disabled people who can work and last but not least, solidarity and love from family and obviously, empathy for all caregivers involved in this long and "windy" road.

Considering the big differences in the necessary endeavors, an acceptable definition of the special needs regarding health care, should consider in an aimed individual, the presence of „functional limitations, linked services health needs and a present state with a minimum current state of standby (for example, 12 months)" (3) - but without the existence of a "gold standard" in the field.

It should be emphasized, an age-related debate: the differentiation - recently noted by the World Health Organization (WHO) - between habilitation (supporting those who obtain impairments congenitally or early in life in order to enhance their skills and functioning so they can develop through training or treatment independence in activities of daily living) and rehabilitation (supporting those who are facing temporary or permanent loss in functioning, later in life). (5)

Under these conditions, the post TBI patients represent a morbidity domain prone to be systematized including through the (new) vision of the WHO - the „International Classification of Functioning, Disability and Health" (ICF-DH) published in 2001, (6) because to the severe neurological challenged people can occur the biggest, harshest and most complex suffering and disabilities.

A very important feature of the ICF-DH is that it allows to be addressed to all matters relating to human health and well-being described as part of health areas such as sight, hearing, walking, learning and memory and related fields health as transport, education and social interactions, within a balanced fit between the medical and respectively, social, models embedded - on which is based this holistic paradigm. (6)

Strong evidence on the importance of the approached subject is that in 2014 it has been developed by the WHO a comprehensive plan sustaining persons with disabilities in the period 2014-2021, with improved health measures for all necesitating people: an approach that seems a significant impulse for the WHO affiliated state governments, within the United Nations, to improve strongly the supporting policies in or- 
der to increase the quality of life (QoL) for impaired people (estimated at 1 billion all over the world), making thus fighting against disabilities a high priority. (7)

Actually within the respective action plan, there are not completly new/unknoun by now items, but just a consistant sistematization within a structured strategy; and this can be determined if recording the main human needs of $\mathrm{V}$. Henderson. By this briliant nurse and academic, essential for proper care, are the human needs related to basic functions like: breathing, feeding and hydration ability, excretion, mobilizing and maintaining postures, sleeping, dressing, maintaining the temperature within normal limits, washing and grooming, preventing dangers, communication, faith, fulfillment, recreation, learning, use of facilities. (8)

\section{APPROACHES AIMING TO IMPROVE POST TBI MEDICAL OUTCOMES}

As known lesions to the brain are among the most likely to cause death or permanent disability. Though some individuals with moderate/ severe brain injury encounter light long-term difficulties, others may demand care or special services for the rest of their lives. (9)

In an effort to improve clinical results after $\mathrm{TBI}$, some scientific institutions have initiated an international multidisciplinary research (in particular the: Canadian Institutes of Health Research, National Institutes of Health and European Commission) have recently collaborated to finance and promote research in TBI, given that, such patients continue to experience including persistent cognitive and emotional disorders more than 5 years after trauma, sequel that tend to evolve in relation to the severity of TBI. (10)

In Romania there are 9 major regional centers specializing in the treatment of neurorehabilitation of patients with severe posttraumatic neuraxial injuries, post acute and chronic stages, of which 4 in universities centers - Bucharest, Cluj, lasi, Timisoara - and 2 in resorts of prime importance: Felix/ 1 Mai, Techirghiol (11). The oldest (celebrating forty years in 2015) and most known of them, including internationally, is the Physical (neural-muscular) and Rehabilitation Medicine Clinic Division of the Teaching Emergency Hospital "Bagdasar Arseni“, in Bucharest.

A health care team composed of physicians (physiatrist, neurologist), psychologist/neuro- psychologist, sociologist, social worker, (kinesy) therapist, physiotherapist, occupational therapist (ergo-therapist), speech language pathologist or (logopedist), general nurses, is dedicated to help an individual after TBI. Family, friends and the involved person are key members of the health care team to work for a common benefit: eventually returning to an as close as possible QoL as previous to the injury. In most cases the effects of TBI lead to changes in roles and responsibilities within the family. Kin and close acquaintances are in a position to fight themselves with behavior changes in such individuals. One frequent modification is that someone in the family becomes a caregiver. (9)

From a medical therapeutic point of view, important targets are severe and moderate TBI's where there can be identified real, specific post TBI consequences (impairments/disabilities) to approach achievable rehabilitative goals.

As including recently systematized in the literature, the main rehabilitative care and nursing approaches recommended and applied in subacute/subchronic patients with severe and even moderate cerebral injuries in order to prevent complications, but not exclusively, are (11):

- regular inspection of the skin for prevention of bedsores; sores are generally preventable when care is carefully applied, but once appeared, it could lead to pain, infections, increased disability and hospitalization period;

- turning (rhythmic change of position) in bed (from 2 to 2 hours if the patient does not have an anti decubitus mattress respectively 4-6 hours if the patient has one;

- anticipatory or corrective posture to restore the anatomical position/function, including using orthotics;

- antideclive limb posture (with role of veno-lymphatic stasis deep thrombophlebitis and respectively, pulmonary thromboembolism prevention);

- passive mobilizations of the paralyzed limb (to improve tissue trophicity and to prevent and/or combat stiffness, also beneficial in terms of kinesthetic memory/representation of the segmental image in the body scheme from the central level;

- procedures of bronchial drainage - possibly if necessary, oxygenotheraphy and/or thoracic tapping); aspiration of the tracheal secretion, all to maintain a healthy respiratory function; 
- assisting/attempt to restore the automatic functions of bladder and bowel;

- a rehabilitative care approach useful for combating sleep disorders can be to keep awake during the day, the post TBI patient through various activities to empower his attention and interest, to be able to sleep easier at night, at bedtime, thus eliminating the known tendency to sleep-wake rhythm disturbance.

As the patient favorably progresses, the cares/rehabilitation nursing role diminishes and the properly rehabilitative programs are growing and hence their importance.

To approach efficiently this complex and difficult pathology, it is necessary to have a "continuum" of the overlapped medical, surgical and respectively rehabilitative services, starting from the (poly) trauma place, the transport to the emergency room, then to the neurosurgery department and possibly other surgical or intensive care units and to the neurorehabilitation unit within the emergency hospital, in the subacute stage, as early as possible. If evolution is favorable, patients can be taken in an „ordinary" postacute/sub-chronic rehabilitation unit - or if the pathology is persistent (marked disability) are granted long-term care/(possibly institutionalization). Home returning is marked by the attempt to reintegrate the patient into family and community as complete as possible, maintaining medical and social attention on the case through the territorial hospitalization and continuations of evaluative, prophylactic, therapeutic, rehabilitative approaches by ambulatory, possibly through balneary cures in appropriate resorts, annually or twice/year and if necessary, by providing socio-economic facilities, legally provided, for people with disabilities. (11-13)

The entire rehabilitative assistance for these patients should be conducted even before the admission to a hospital unit and should continue as long as it is necessary. (13)

\section{CONCLUSIONS}

The tracked points in the rehabilitation of patients with moderate/severe TBI are to reduce the rate of functional sequel and cognitive disorders, also of complications, to achieve - if the case - new skills with functional objectives based on remaining structures or functions after injury and when needed to provide with appropriate assistive devices and knowledge in order to optimally used them, to achieve independence in self-care and to obtain a better QoL, comprising good family relationships and social reintegration, including through professional based advocacy to which this paper might hopefully give a modest contribution.

\section{$\overline{\text { REFERENCES }}$}

1. http://www.who.int/topics/disabilities/en/

2. Muresanu D.F. Neuroprotection and neuroplasticity: two aspects of a continuous process, genetically regulated and powered by neurotrophic factors. Communication at the 6th AMN Congress, Cluj- Napocal Romania, 2008

3. Bethell C., Read D. et al. Approaches to Identifying Children and Adults with Special Health Care Needs (A Resource Manual for State Medicaid Agencies and Managed Care Organizations). Prepared by CAHMI - The Child and Adolescent Health Measurement Initiative. On behalf of the Centers for Medicare and Medicaid Services. The Foundation for Accountability (FACCT), 2002 \& Oregon Health \& Science University; http://www.cahmi.org/ wp-content/uploads/2014/06/CSHCNSCMS-manual.pdf

4. Huntington B., Swanson C., Burmaster E. Adults with Special Needs A Resource and Guide for Wisconsin's Public Libraries (2000 to 2002)
5. http://who.int/disabilities/policies/actionplan/ disability_action_plan_en.docx

6. WHO: ICF International Classification of Functioning Disability and Health. Geneva, World Health Organization, 2001 - http:// www.handicapincifre.it/documenti/icf_18. pdf; http://search.tb.ask.com/search/ GGmain.jhtml?searchfor=WHO\%3A + ICF+International + Classification of+ Functioning+Disability+and+Health.+\&tpr= hpsb\&p2=\%5EBBQ\%5Exdm027\% $5 E S 11598 \% 5$ Ero\&n=780CE8B2\&st= hp\&ptb=DB405F6C-8D31-4124-B4562183793D26CA\&qs=\&si= downspeedtest\&ts $=1422908344825$

7. http://www.who.int/mediacentre/ events/2014/wha67/en/

8. http://nursing-theory.org/nursing-theorists/ Virginia-Henderson.php

9. Understanding Brain Injury. A Guide for the Family. Page 1 of 34 MC1298-01

10. Sharma B., Lawrence D.W. Top-Cited Articles in Traumatic Brain Injury. Front Hum Neurosci. 2014; 5:8-879.
11. Onose G. Cap.: Noțiuni sintetice de management neuroreabilitativ în statusuri post traumatisme cranio-cerebrale. In: Popescu I. Ciuce C. Tratat național de chirurgie. Florian. I. St. Poeata I. Vol. VI. Neurochirurgie: Editura Academiei Române, 2015: 440-473

12. Boake C., Francisco G.E., Ivanhoe C.B., Kothari S. Brain Injury Rehabilitation - in: Braddom R.L. et al. - Physical Medicine \& Rehabililitation (2nd edition). W.B. Saunders Company, Philadelphia, U.S.A., 2000

13. von Wild K. RH. Early rehabilitation of higher cortical brain functioning in neurosurgery, humanizing the restoration of human skills after acute brain lesions. Acta Neurochir. Suppl. 2006; 99:3-10 